OPEN ACCESS

Edited by:

Ashok Kumar,

University of Florida, United States

Reviewed by:

Craig Stark,

University of California, Irvine,

United States

Michael John O'Sullivan

The University of Queensland,

Australia

*Correspondence:

Dayana Hayek

dayana.hayek@gmail.com

Daria Antonenko

daria.antonenko@uni-greifswald.de

Received: 26 October 2019

Accepted: 04 March 2020

Published: 20 March 2020

Citation:

Hayek $D$, Thams F, Flöel $A$ and Antonenko D (2020) Dentate Gyrus Volume Mediates the Effect of Fornix Microstructure on Memory Formation

in Older Adults.

Front. Aging Neurosci. 12:79

doi: 10.3389/fnagi.2020.00079

\section{Dentate Gyrus Volume Mediates the Effect of Fornix Microstructure on Memory Formation in Older Adults}

\author{
Dayana Hayek ${ }^{1,2 *}$, Friederike Thams ${ }^{2}$, Agnes Flöel ${ }^{1,2,3}$ and Daria Antonenko ${ }^{1,2 *}$ \\ 1 Department of Neurology, NeuroCure Clinical Research Center, Berlin Institute of Health, Corporate Member of Freie \\ Universität Berlin, Charité - Universitätsmedizin Berlin, Humboldt-Universität Berlin, Berlin, Germany, ${ }^{2}$ Department \\ of Neurology, Universitätsmedizin Greifswald, Greifswald, Germany, ${ }^{3}$ German Centre for Neurodegenerative Diseases \\ (DZNE) Standort Greifswald, Greifswald, Germany
}

Age-related deterioration in white and gray matter is linked to cognitive deficits. Reduced microstructure of the fornix, the major efferent pathway of the hippocampus, and volume of the dentate gyrus (DG), may cause age-associated memory decline. However, the linkage between these anatomical determinants and memory retrieval in healthy aging are poorly understood. In 30 older adults, we acquired diffusion tensor and T1-weighted images for individual deterministic tractography and volume estimation. A memory task, administered outside of the scanner to assess retrieval of learned associations, required discrimination of previously acquired picture-word pairs. The results showed that fornix fractional anisotropy (FA) and left DG volumes were related to successful retrieval. These brain-behavior associations were observed for correct rejections, but not hits, indicating specificity of memory network functioning for detecting false associations. Mediation analyses showed that left DG volume mediated the effect of fornix FA on memory (48\%), but not vice versa. These findings suggest that reduced microstructure induces volume loss and thus negatively affects retrieval of learned associations, complementing evidence of a pivotal role of the fornix in healthy aging. Our study offers a neurobehavioral model to explain variability in memory retrieval in older adults, an important prerequisite for the development of interventions to counteract cognitive decline.

Keywords: aging, brain plasticity, white matter integrity, hippocampal subfields, tractography

\section{INTRODUCTION}

Age-related deterioration of white and gray matter in the human brain contributes to cognitive impairment in the course of aging (Grady, 2012; Marstaller et al., 2015). Microstructure in white matter tracts as assessed by diffusion tensor imaging (DTI) and gray matter volume estimated from high-resolution structural magnetic resonance images predict behavioral task performance in healthy older adults (Antonenko et al., 2012; Sasson et al., 2013). Episodic memory, as one of the most vulnerable cognitive domains in aging (Nyberg et al., 2012), is mediated by hippocampal networks, including the hippocampus itself and the fornix as its major efferent white matter pathway (Metzler-Baddeley et al., 2011; Lovblad et al., 2014; Antonenko et al., 2016b; Gorbach et al., 2017; Anblagan et al., 2018). 
Reduced fornix white matter microstructure has been observed in the course of healthy, but also pathological aging processes (Metzler-Baddeley et al., 2012; Pelletier et al., 2013; Zhuang et al., 2013). Deteriorated fornix pathways were suggested to be one of the earliest abnormalities in older individuals with mild cognitive impairment (MCI) who progress to Alzheimer's dementia (AD) (Douaud et al., 2013; Kantarci, 2014). Inter-individual variability in fornix macro- and microstructure has been associated with older adults' variability in performance on various episodic memory tasks that require verbal or visual recall or recognition of items and associations (Metzler-Baddeley et al., 2011; Lockhart et al., 2012; Douet and Chang, 2014; Henson et al., 2016; Gazes et al., 2018). Likewise, the hippocampus is susceptible to healthy and pathological aging, leading to altered episodic memory function (Frisoni et al., 2008; Walhovd et al., 2011; den Heijer et al., 2012). Whether or not volumetric atrophy of the whole hippocampus contributes to age-related memory decline is unclear (Head et al., 2005; Fjell et al., 2010; Pereira et al., 2014). This may be due to differential vulnerability of its subfields to aging (Small et al., 2002, 2011). Within the hippocampal formation, advanced age has been associated mainly with reduced volumes in its subfields cornu ammonis 1-4 and dentate gyrus (DG) (Mueller and Weiner, 2009; Pereira et al., 2014). The DG was observed to have a stronger age-associated cerebral blood volume decrease compared to other hippocampal subfields (Brickman et al., 2014). Due to its particular vulnerability to age-related processes (Small et al., 2004; Wilson et al., 2006) and activity during memory discrimination tasks, changes in DG has been suggested to drive age-related cognitive decline (Yassa et al., 2011a,b; Doxey and Kirwan, 2015).

Of particular importance in the context of healthy aging and $\mathrm{AD}$ is the question of mutual dependency of gray and white matter damage, i.e., their directional relationship and interaction to predict cognitive decline (cf. Bartzokis, 2011; Dansokho and Heneka, 2018; Metzler-Baddeley et al., 2019). Within structural hippocampal networks, correlational studies have found positive associations between hippocampal atrophy and loss of fornix connections in older adults, with findings pointing toward a pivotal role of the latter in older age (Fletcher et al., 2013; Pelletier et al., 2013; Zhuang et al., 2013; Gazes et al., 2018). Using mediation analyses, a recent study applied individual white matter fiber tractography and hippocampal segmentation to investigate the linkage between increased age, reduced fornix microstructure and hippocampal atrophy in a directional approach (Metzler-Baddeley et al., 2019). The results revealed that white matter changes predicted gray matter deterioration, but not vice versa, concordant with the idea of age-related myelin damage causing abnormal intracellular metabolism and neuronal death (Bartzokis, 2004; Metzler-Baddeley et al., 2019). Even with evidence from previous research showing a structural connectivity between fornix and subiculum (Saunders and Aggleton, 2007), animal studies have observed a unique functional connection between the fornix and the DG subfield (Gondard et al., 2015; Hao et al., 2015). The authors showed that deep brain stimulation of the fornix activated the DG by modulating the expression of neurotrophic factors and markers of synaptic plasticity known to be crucial for memory processing. These studies could indicate that disconnection in forniceal white matter pathways might induce functional and/or structural changes in hippocampus, and more specifically in its DG subfield, which in turn may affect age-related decline in memory function. The interactive effect of both structures on the ability to form novel memories in older adults has not been elucidated yet. In the present study, we aimed to investigate this linkage between structural hippocampal networks and the retrieval of episodic memory in healthy older adults. We administered a task that required learning of new picture-word associations and subsequent discrimination of correct and incorrect pairings during retrieval in order to assess hippocampus-dependent memory performance (Antonenko et al., 2016a, 2019). Individual forniceal pathways were reconstructed on diffusion-weighted images using deterministic tractography based on the constrained spherical deconvolution (CSD) technique. Based on previous findings suggesting the involvement of left hippocampus and its subfields in similar or the same verbal memory tasks (Breitenstein et al., 2005; Pereira et al., 2014; Witt et al., 2014; Doxey and Kirwan, 2015; Powell et al., 2016; De Shetler and Rissman, 2017; Antonenko et al., 2019), individual volumes of the left DG were estimated on T1 images using automated subcortical segmentation (Iglesias et al., 2015). We aimed to explore correlational relationships between memory retrieval performance in different response categories reflecting the detection of correct and incorrect associations, fractional anisotropy (FA) in the fornix and volume in the left DG. Subsequent mediation models were conducted to evaluate the linkage between structural properties and memory performance.

\section{MATERIALS AND METHODS}

\section{Participants and Study Design}

Thirty healthy older subjects between the age of 50 and 79 years were recruited in this study (14 f; mean/SD age: 62/6 years). They were all right-handed, German native speakers, with no history of neurological diseases. Neuropsychological testing was performed for all participants to assure normal cognitive functioning within age- and education-related norms (CERAD-Plus) $)^{1}$ (Table 1). The study was approved by the Ethics Committee of the Charite University Medicine and conducted in accordance with the Helsinki Declaration. Written informed consent was obtained from all participants prior to participation.

\section{Episodic Memory Task}

The task was adapted from previous studies (Breitenstein and Knecht, 2002; Breitenstein et al., 2005; Floel et al., 2008; Antonenko et al., 2016a, 2019), and programmed using the software Presentation (Neurobehavioral Systems) ${ }^{2}$. The paradigm consists of the presentation of 30 picturepseudoword pairs. For each participant, a set of 30 pseudowords and 30 pictures of daily life were randomly matched to

\footnotetext{
${ }^{1}$ https://www.memoryclinic.ch/de/

${ }^{2}$ http://www.neurobs.com/, version 18.1
} 
TABLE 1 | Characteristics of participants.

\begin{tabular}{|c|c|c|}
\hline & Mean & SD \\
\hline Age, years & 62 & 6 \\
\hline Education, years & 15 & 2 \\
\hline $\mathrm{LQ}^{\mathrm{a}}$ & 94.3 & 9.3 \\
\hline $\mathrm{GDS}^{\mathrm{b}}$ & 1.3 & 1.4 \\
\hline \multicolumn{3}{|l|}{ Digit Span (max. 14) } \\
\hline Forward & 7.6 & 2.4 \\
\hline Backward & 6.1 & 1.9 \\
\hline Vocabulary test $(\max .37)^{\mathrm{C}}$ & 33.2 & 2.1 \\
\hline Semantic fluency, N (in 60s) & 25.3 & 5.6 \\
\hline Boston Naming Test, N (max.15) & 14.7 & 0.5 \\
\hline Mini-Mental State (max. 30) & 29.4 & 0.9 \\
\hline \multicolumn{3}{|l|}{ Word list learning, $N$} \\
\hline Total (max. 30) & 23.3 & 3.0 \\
\hline Trial 1 (max. 10) & 6.2 & 1.5 \\
\hline Trial 2 (max. 10) & 8.1 & 1.3 \\
\hline Trial 3 (max. 10) & 9.1 & 0.9 \\
\hline Word list retrieval, $N$ (max. 10) & 8.3 & 1.2 \\
\hline Word list intrusions, $N$ & 1.0 & 2.0 \\
\hline Figure copying, $N(\max .11)$ & 11 & 0.0 \\
\hline Figure retrieval, $N(\max .11)$ & 10.7 & 0.8 \\
\hline Phonemic fluency, $N$ (in 60s) & 16.1 & 4.1 \\
\hline \multicolumn{3}{|l|}{ Trail making test, $s$} \\
\hline Part A & 38.9 & 10.7 \\
\hline Part B & 79.0 & 20.2 \\
\hline
\end{tabular}

generate 30 "correct" picture-pseudoword combinations (e.g., elephant = "pari"), creating a "vocabulary" list which had to be learned over the course of five blocks and retrieved in a "transfer" block (where pictures were replaced by corresponding German words). "Incorrect" pairs (e.g., elephant = "ralm") were created by combining a picture with other pseudowords from the set. The task was originally created to resemble natural language learning, so the underlying learning principle involves the detection of higher co-occurrences of "correct" compared to "incorrect" pairs (Breitenstein and Knecht, 2002).

During the learning phase, 600 trials were presented, divided into five blocks (120 trials per block). "Correct" pairings were presented ten times in total (i.e., twice in each block, totaling up to 60 "correct" pairs per block). Each of the 30 pictures was also presented ten times with varying "incorrect" pseudowords (i.e., each picture was paired with two different "incorrect" pseudowords per block, totaling up to 60 "incorrect" pairs per block). Each "incorrect" pairing was presented only once over the course of the whole task. Trial presentation order was randomized. Participants were instructed to answer as quickly as possible by button press if the pairing was "correct" or not, being however not informed about the underlying frequency principle. No feedback was given during the task. As the learning principle of the task is based on the detection of higher cooccurrences of (arbitrarily) "correct" compared to "incorrect" couplings (ratio 10:1) over the course of the five learning blocks, participants had to guess in the beginning of the task. In each trial of the learning phase, the picture was presented $200 \mathrm{~ms}$ after the onset of an auditory spoken pseudoword (normalized at the same loudness and length of $600 \mathrm{~ms}$, delivered over headphones) and remained on the screen for $1500 \mathrm{~ms}$. Performance accuracy increased from first to last learning block, indicating that participants were able to learn the pairings (repeated-measures ANOVA showed a significant main effect of learning blocks on performance accuracy $[F(2.5,71.0)=74.43, p<0.001$, partial eta squared $=0.72$ ), see Supplementary Figure $\mathbf{S} 1$ for individual learning curves).

During the retrieval block, corresponding spoken German words were presented instead of pictures together with the pseudowords. Stimulus count, underlying frequency principle (i.e., 60 "correct" and 60 "incorrect" pairings; the latter containing again different pseudowords than in the learning phase) and trial timings were identical to those in a learning block. Total duration of the task was $35 \mathrm{~min}$. The task generated four response categories: hits (i.e., classifying a "correct" pair as "correct"), correct rejections (i.e., classifying an "incorrect" pair as "incorrect"), false alarms (i.e., classifying an "incorrect" pair as "correct"), and misses (i.e., classifying a "correct" pair as "incorrect") (Lynn and Barrett, 2014). Distribution of performance in response categories was illustrated in Supplementary Figure S2. Percentage of total correct responses and percentage of correct responses in different response categories were examined in the retrieval phase.

\section{MRI Acquisition}

MRI was performed using 3T Siemens Trio MR-System using 12-channel head coil at the Berlin Center for Advanced Neuroimaging. First, a 3D structural highresolution T1-weighted magnetization prepared rapid gradient echo image was acquired with the subsequent characteristics; TR $=1900 \mathrm{~ms}, \mathrm{TE}=2.52 \mathrm{~ms}, 192$ sagittal slices, voxel size $=1.0 \mathrm{~mm}^{3} \times 1.0 \mathrm{~mm}^{3} \times 1.0 \mathrm{~mm}^{3}$, flip angle $=9^{\circ}$. Second, a diffusion-weighted spin-echo echoplanar imaging image was acquired with the subsequent characteristics; TR $=7500 \mathrm{~ms}$, TE $=86 \mathrm{~ms}, 61$ axial slices, voxel size $=2.3 \mathrm{~mm}^{3} \times 2.3 \mathrm{~mm}^{3} \times 2.3 \mathrm{~mm}^{3} ; 64$ directions, $b$-value of $1000 \mathrm{~s} / \mathrm{mm}^{2}, 1 \mathrm{~b} 0$.

\section{MRI Data Analysis}

\section{Tractography and Tract Variables}

Tractography was performed using ExploreDTI (Leemans et al., 2009). Diffusion MRI images were corrected for eddy currents and distortions caused by head motion. Metzler-Baddeley et al. (2011) previously described that diffusion tensor model tractography (Basser et al., 2000) was not found to be the most appropriate technique to reconstruct the fornix because it is positioned next to other white matter tracts. Thus, we used deterministic tracking based on CSD which was shown to resolve crossing fibers problem (Tournier et al., 2004, 2007, 2008). At each voxel, CSD extracts peaks in the fiber orientation density function (fODF). The fODF estimates the proportion of fibers in a voxel pointing in each direction. The diffusion orientation was estimated at each seed point and then moved further in 
$0.5 \mathrm{~mm}$ steps along the direction that traversed the smallest angle of the trajectory. A pathway was depicted until either a change in direction with an angle $>60^{\circ}$ or a drop of FA value below 0.2 occurred (Carballedo et al., 2012; Antonenko et al., 2016b). Whole brain tractography was performed using every voxel as seed point. In order to extract three-dimensional reconstructions of the fornix and the uncinate fasciculus, multiple way-point regions of interest (ROIs) masks were implemented. This technique uses Booleian logical operations to delineate ROIs specific masks, for instance, one can choose to reconstruct a tract that passes through ROI-1 but NOT ROI2. Following training, all ROIs were manually drawn in native space using color-coded fiber orientation maps for individual subjects. This was performed by a single operator blinded to age, neuropsychological data and memory performance, using landmark techniques that have been defined in previously published studies and that have been shown to be highly reproducible, valid and reliable (Catani et al., 2002; MetzlerBaddeley et al., 2011; Carballedo et al., 2012), see Figure 1. The main outcome measure was mean FA. FA is one DTI variable that measures the degree of directionality of the diffusion in a specific white matter structure, and it is calculated from the diffusion tensor eigenvalues that give the directions of the diffusion (Basser and Pierpaoli, 1996). For white matter pathways, FA is the most widely used measure to examine microstructure and integrity (Alexander et al., 2007; Zatorre et al., 2012).

In order to delineate the individual tracts, first, medial level of a coronal section where the anterior pillars enter into the body of the fornix was located. "SEED" point ROI was drawn around the body of the fornix bundle. Second, at the inferior part of the splenium of the corpus callosum, "AND" ROI around the crus fornici of each hemisphere was drawn. Finally, to eliminate unwanted tracts, three "NOT" ROIs were drawn; one is rostral to the fornix pillars; one is caudal to the crus fornici, and a third one on an axial slice through the upper pons and the corpus callosum (for individual tracts, see Supplementary Figure S3). The same procedure was used to extract FA values from the uncinate fasciculus that served as a control white matter tract. "AND" ROIs were drawn on the posterior coronal slices on the separation point of temporal and frontal lobe. The first ROI was placed on the temporal lobe and the second ROI on a more caudal coronal section to include the projections toward the frontal lobe. Finally, caudal "NOT" ROI was placed to eliminate irrelevant fibers (for individual tracts, see Supplementary Figure S4). Every individual tract was visually inspected and inadequate outlier tracts were removed using additional "NOT" ROIs.

\section{Hippocampal Subfields Volume}

Individual volumes of the left DG (precisely referring to the left granule cell layer and molecular layer of the DG), left CA1 and left subiculum (which both served as a control hippocampal subfield) were segmented using FreeSurfer (version 6.0) (3) $^{3}$ algorithm, introduced by Iglesias et al. (2015). Fully automated cortical and subcortical reconstructions and volumetric segmentations, including the hippocampus were performed (for individual

${ }^{3}$ http://surfer.nmr.mgh.harvard.edu/

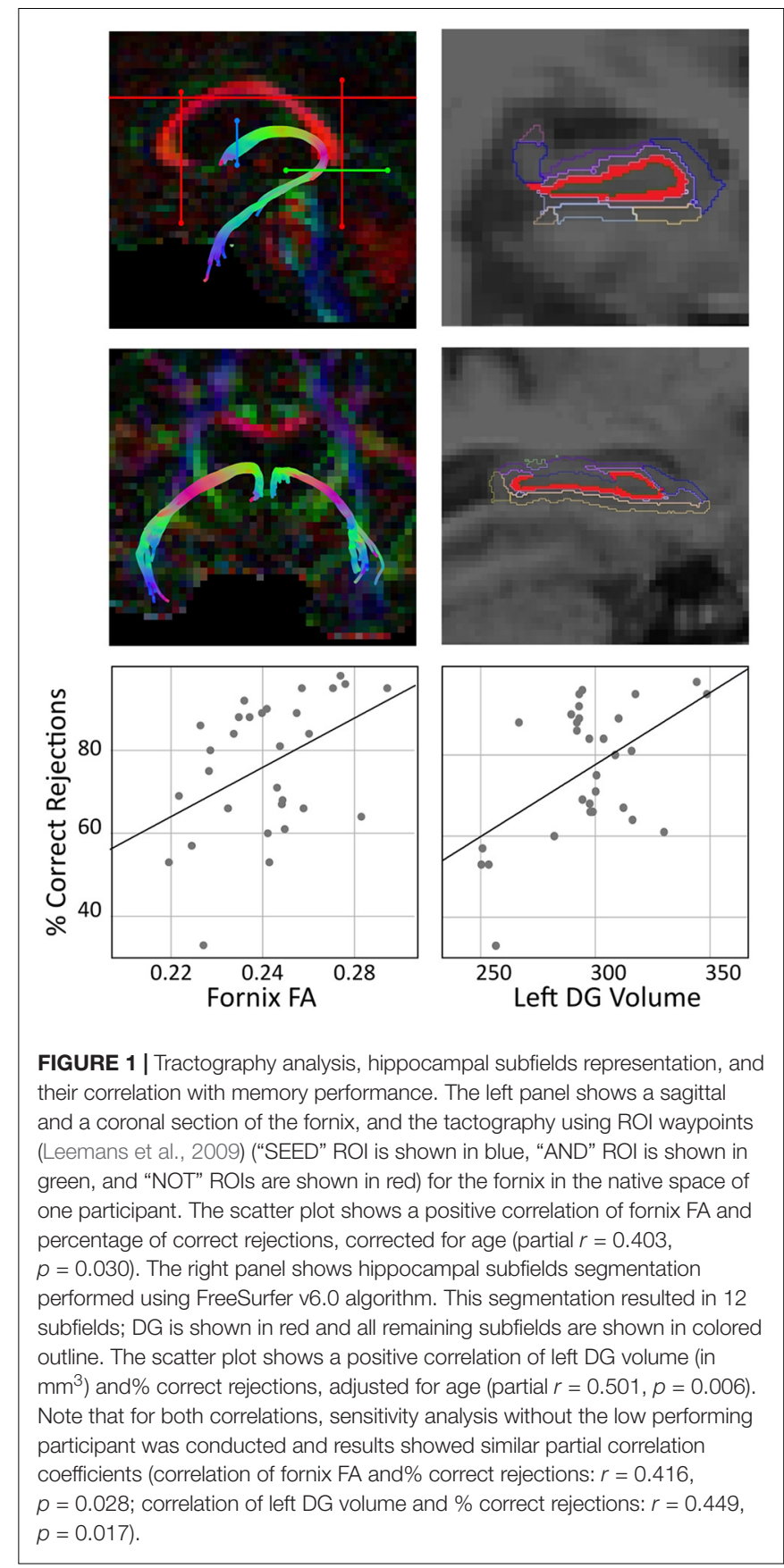

volumetric segmentation of all hippocampal subfields, see Supplementary Figure S5). Preprocessing of T1-weighted images included intensity normalization, skull stripping and automated topology correction using a watershed algorithm (Fischl et al., 2002). Individual left DG, left CA1 and left subiculum volumes were adjusted for intracranial volume (ICV) (den Heijer et al., 2012; Kerti et al., 2013; Kobe et al., 2017) with the following formula:

Adjusted Volume $=$ raw volume $-b \times($ ICV - mean ICV $)$.

The coefficient $b$ indicates the regression slope of the region to be adjusted on the ICV. Individual hippocampal subfields were 
superimposed on anatomical images. Segmentation quality was visually inspected and no further correction was necessary.

\section{Statistical Analysis}

We used SPSS $25.0^{4}$ to perform all statistical analyses. Partial correlation coefficients were computed for correlation analyses of brain structural variables and behavioral performance, corrected for age. As per Hartopp et al. (2018), partial correlations with hippocampal subfields, white matter microstructures and memory performance were performed. We implemented a simple mediation analysis using PROCESS (Hayes and Preacher, 2014; Zamroziewicz et al., 2017; Zhang et al., 2018) in order to test whether the relation between a predictor (fornix FA) and an outcome (percentage of correct rejections) is mediated - in total or in part - by a mediator variable (volume of left DG, and as control regions; volume of left CA1 or left subiculum) (Baron and Kenny, 1986). We assessed the indirect effect of the independent variable (IV) and the dependent variable (DV) through a mediator variable (MV), corrected for age. To test this hypothesis, a bootstrapping resampling strategy was implemented while taking 5000 bootstrap samples. Path $a$ describes the direct effect of the IV (fornix FA) on the MV (left DG volume; volume of left CA1 or left subiculum), path $b$ represents the direct effect of the MV (left DG volume; volume of left CA1 or left subiculum) on the DV (percentage of correct rejections), and path $c$ indicates the total effect of IV and MV on the DV. Finally, path $c^{\prime}$ reveals the direct effect of the IV (minus MV) on the DV (Figure 2 and Supplementary Figure S6). Bias-corrected 95\% confidence interval (CI) was computed to evaluate the contribution of the $\mathrm{MV}$ (indirect effect, path $\mathrm{a} \times \mathrm{b}$ ). CI reached significance when the interval range did not include zero.

\section{RESULTS}

\section{Memory and Fornix White Matter Microstructure}

Fornix FA showed a positive correlation with percentage of total correct responses (partial correlation coefficient: $r$ (corrected for age) $=0.39, p=0.035$ ). In order to examine whether this relationship was specific to a response category, we further examined separate correlations between Fornix FA and percentage of hits and correct rejections: Fornix FA did not correlate with percentage of hits (partial $r=-0.001, p=0.995$ ) but showed a positive correlation with percentage of correct rejections (partial $r=0.403, p=0.030$, Figure 1). FA values extracted from the uncinate fasciculus did not correlate with percentage of correct rejections (partial $r=0.299, p=0.115$, see Supplementary Figure S7).

\section{Memory and Left DG Volume}

Left DG volume showed no strong correlation with percentage of total correct responses (partial correlation coefficient: $r$ (corrected for age) $=0.291, p=0.126)$. In order to explore this relationship in different response categories, we further examined

${ }^{4} \mathrm{http} / / / \mathrm{www}-01 . i b m . c o m / s o f t w a r e / u k / a n a l y t i c s / s p s s /$

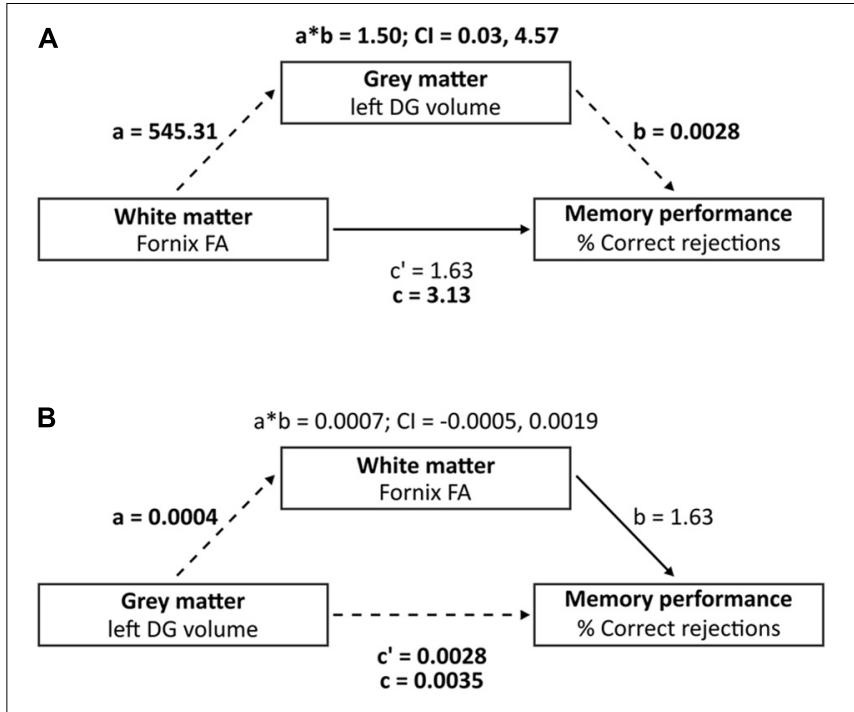

FIGURE 2 | Mediation analysis investigating the three-way association between left DG volume, Fornix FA and percentage of correct rejections. (A) Mediation analysis (model A) where "a" indicates the effect of fornix FA on left DG volume, "b" indicates the effect of left DG volume on \% correct rejections, adjusted for fornix FA, i.e., direct effect of left DG volume on \% correct rejections, "c" indicates the direct effect of fornix FA on \% correct rejections, and "C" indicates the total effect (direct and indirect) of fornix FA on $\%$ correct rejections. (B) Reverse mediation analysis (model B) where "a" indicates the effect of left DG volume on fornix FA, "b" indicates the effect of fornix FA on \% correct rejections, adjusted for left DG volume, i.e., direct effect of fornix FA on \% correct rejections, "c" indicates the direct effect of left DG volume on \% correct rejections, and "c" indicates the total effect (direct and indirect) of left DG volume on \% correct rejections. Dashed lines indicate significant paths and bold font indicates significant terms. Bias-corrected 95\% $\mathrm{Cl}$ is displayed for the indirect effects $\left(\mathrm{a}^{*} \mathrm{~b}\right)$.

separate correlations between left DG volume and percentage of hits and correct rejections: left DG volume did not correlate with percentage of hits (partial $r=-0.133, p=0.492$ ) but showed a positive correlation with percentage of correct rejections (partial $r=0.501, p=0.006$, Figure 1). Volume left CA1 did not correlate with percentage of correct rejections (partial $r=0.341$, $p=0.071$, see Supplementary Figure S7). Correlations with all other hippocampal subfields were statistically non-significant (Supplementary Table S1, all p's > 0.05).

\section{Mediation Model Analysis}

Our model including fornix FA, memory performance and left DG volume met the criteria for mediation, where path $a, b$ and $c$ showed significant associations $(p<0.05)$ and path $c^{\prime}$ did not show significant associations (Table 2). Single mediation analysis (model A) showed an indirect effect of fornix FA on percentage of correct rejections, mediated by left DG volume, corrected for age $(\beta=1.50,95 \%$ CI: $0.03,4.57$, Table 2 and Figure 2A). The mediation effect (path ab) constituted $48 \%$ of the total effect of fornix FA on percentage of correct rejections (path c). Reverse mediation analysis (model B) was performed to investigate whether fornix FA mediates the effect of left DG volume on percentage of correct rejections, as such, we can induce the specificity of left DG volume in mediating fornix 
TABLE 2 | Mediation analysis models of the three-way association between left DG volume, Fornix FA, and percentage of correct rejections ( $n=30)$.

\begin{tabular}{|c|c|c|c|c|}
\hline Effect & Coefficient \pm SE ( $\%$ Mediation) & $t$ & $p$ & $95 \% \mathrm{Cl}$ \\
\hline \multicolumn{5}{|l|}{ Model A } \\
\hline Total effect c (fornix FA on \% correct rejections) & $3.13 \pm 1.37$ & 2.28 & $0.030^{\star}$ & \\
\hline a (fornix FA on left DG volume) & $545.31 \pm 189.80$ & 2.87 & $0.008^{\star \star}$ & \\
\hline b (left DG volume on \% correct rejections) & $0.0028 \pm 0.0013$ & 2.10 & $0.045^{\star}$ & \\
\hline Mediation effect ab (fornix FA on \% correct rejections via left DG volume) & $1.50 \pm 1.03(48)$ & - & - & $0.03,4.57$ \\
\hline Direct effect c' (fornix FA on \% correct rejections) & $1.63 \pm 1.47$ & 1.10 & 0.279 & \\
\hline \multicolumn{5}{|l|}{ Model B } \\
\hline Total effect c (left DG volume on \% correct rejections) & $0.0035 \pm 0.0012$ & 3.0 & $0.006^{\star \star}$ & \\
\hline a (left DG volume on fornix FA) & $0.0004 \pm 0.0001$ & 2.87 & $0.008^{* *}$ & \\
\hline b (Fornix FA on \% correct rejections) & $1.63 \pm 1.47$ & 1.10 & 0.279 & \\
\hline Mediation effect ab (left DG volume on \% correct rejections via Fornix FA) & $0.0007 \pm 0.0006$ & - & - & $-0.0005,0.0019$ \\
\hline Direct effect c' (left DG volume on \% correct rejections) & $0.0028 \pm 0.0013$ & 2.10 & $0.045^{\star}$ & \\
\hline
\end{tabular}

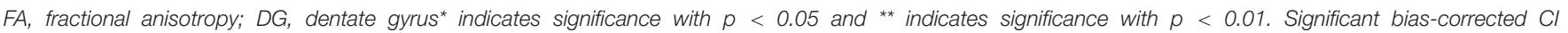
are shown in bold.

FA effect of percentage of correct rejections. Reverse mediation analysis showed that fornix FA did not mediate the effect of left DG volume on percentage of correct rejections, corrected for age ( $\beta=0.0007,95 \% \mathrm{CI}:-0.0005,0.0019$, Table 2 and Figure 2B). These findings support the mediation effects of left DG volume on the relationship between fornix FA and percentage of correct rejections. Further mediation analysis showed that neither left CA1 nor left subiculum mediated the effect of fornix FA on the percentage of correct rejections (Supplementary Figure S6).

\section{DISCUSSION}

The current study investigated the effect of fornix white matter microstructure and left DG volume on the retrieval of episodic memory in healthy older adults. Fornix FA and left DG volume were correlated with correct rejections (i.e., successful retrieval of "incorrect" associations) during retrieval of previously acquired picture-word pairs. Mediation analysis further showed that the prediction of memory performance by increased fornix microstructure was mediated by higher volume in the left DG, but not vice versa. This finding indicates that reduced fornix microstructure impairs successful retrieval of learned associations through its impact on left DG subfield of the hippocampus.

\section{Association of Fornix White Matter Microstructure and Memory Performance}

Our finding of a positive association between fornix FA and memory performance is in line with previous studies in both young and older adults (Rudebeck et al., 2009; Douet and Chang, 2014; Ly et al., 2016). Ly et al. (2016) found that variability in fornix microstructure in middle-to-late aged adults was related to face recognition memory and partly explained preserved functional connectivity within hippocampal networks. A study by Metzler-Baddeley et al. (2011) further suggested that specifically age-related degradation of fornix microstructure as derived from individual fiber tracking was linked to memory recall performance in strategic and visual memory tasks in older adults (Metzler-Baddeley et al., 2011). Our data confirms the role of the fornix in older adults in verbal episodic memory (MetzlerBaddeley et al., 2011, 2012). Moreover, we found a positive link between fornix FA and percentage of correct rejections in older adults, indicating that preservation of forniceal fiber pathway integrity with increasing age may be crucial for the ability to detect false associations. We found no correlation of FA of the uncinate fasciculus with percentage of correct rejections. Bennett et al. (2015) found effects of fornix FA on the prediction of pattern separation performance. They also found no effect of uncinate fasciculus and cingulum bundle FA on the prediction pattern separation performance. Taken together, these findings suggest that pattern separation performance, corresponding to the ability to detect false associations, relies selectively on fornix microstructural integrity.

\section{Association of Dentate Gyrus Volume and Memory Performance}

In line with previous studies, age-related preservation in left DG volumes was also associated with superior memory performance (Small et al., 2002; Toner et al., 2009; Stark and Stark, 2017; Bennett et al., 2018). Our data further suggests that in particular the inhibition of false memories may be sensitive to the effects of age-related atrophy in the left DG. This result complements the findings of Shing et al. (2011) who showed that DG volumes in healthy older adults were negatively correlated to false alarm rates [which are complementary to correct rejection rates (Lynn and Barrett, 2014)] in a word-pair learning task. The authors hypothesized that the role of the DG is to enhance the specificity of encoded memories. In order to store overlapping inputs, DG performs pattern separation that enables the correct retrieval of interfering information (Rolls, 2010). As such, successful rejection of false associations is based on the efficient representation of differences between the correct and the incorrect associations. Interestingly, older adults show a specific decrease in their pattern separation ability, making them more 
vulnerable to memory distortions (Toner et al., 2009; Stark et al., 2010). It is also possible that this specific association between left DG volume and correct rejections reveals the function of left DG in implementing retrieval strategies to prevent these memory distortions [for review (Kesner et al., 2004)]. Novelty detection is one strategy that has been related to the DG and that allows detection of newly presented information. Our findings lend further support to this concept, assuming that participants may have implemented this strategy to successfully detect new (i.e., incorrect) associations. Additionally, we found no correlation of percentage of correct rejections with left CA1 subfield volume, confirming that this subfield may not be crucial for pattern separation (Bakker et al., 2008). This suggests that our results were specific for the left DG.

\section{Association of Fornix White Matter Microstructure and Memory Performance Was Mediated by Dentate Gyrus Volume}

In the present study, correct rejections, used as main outcome in the mediation analysis, most likely quantified pattern separation performance that has been associated to both, DG and fornix (Shing et al., 2011; Bennett et al., 2015). Combining both white matter microstructure and gray matter volume within the structural hippocampal memory network, our mediation analysis showed that the prediction of memory performance by fornix microstructure was partially mediated by the volume of left DG in older adults. Previous studies have shown a link between age-related decrease of fornix integrity and hippocampal atrophy (Pelletier et al., 2013; Zhuang et al., 2013; Wisse et al., 2015; Gazes et al., 2018). The directional relationship, however, remains unclear, as it is conceivable that both hippocampal gray matter loss induces fornix white matter fiber degeneration and vice versa (cf. Zhuang et al., 2013). So far, it has been shown that fornix microstructural degradation, and not hippocampal atrophy, served as a biomarker for early amnestic MCI due to AD (Zhuang et al., 2013). Further, using mediation analysis, a recent study provided evidence for a causal effect of age-related fornix white matter damage on hippocampal gray matter volume decline in healthy adults (Metzler-Baddeley et al., 2019). The current study complements previous evidence by demonstrating that fornix microstructure and left DG subfield volume interact to specifically mediate memory for false associations.

It should be noted that cells of the DG do not project outside of the hippocampal formation, so this area can provide only indirect input to the fornix (Insausti and Amaral, 2004). However, Gondard et al. (2015) showed that deep brain stimulation of the fornix activated the DG by modulating the expression of neurotrophic factors and markers of synaptic plasticity known to be crucial for memory processing. This association could be explained by the interconnectivity of subfields in the hippocampus, forming a functional unit within the hippocampal formation (cf. Duvernoy et al., 2005; Wisse et al., 2015). Based on this, one can assume that structural proximity between hippocampal subfields may make it difficult to clearly distinguish individual subfield function. Rather, the specificity of hippocampal subfields function may be demonstrated by its exclusive effect on specific cognitive outcomes. In patients with $\mathrm{MCI}$ and $\mathrm{AD}$, in particular atrophy of the subiculum was associated with fornix microstructure (Wisse et al., 2015) which may point toward different patterns of hippocampal disconnection in early AD. Future studies are needed that unveil the interconnectivity between hippocampal subfields and white matter tracts involved in this circuit.

In conclusion, our mediation model offered a neurobehavioral model in which preserved fornix white matter microstructure in older age predicts successful retrieval of learned associations through its protective effect on gray matter volume in the left DG. Thus, demyelination, even in healthy aging, may induce gray matter loss reflecting altered intracellular metabolism or neural death in connected structures, and leading to constrained memory performance (Bartzokis, 2004; Metzler-Baddeley et al., 2019). Due to the crosssectional design of our study that still limits conclusions about causality; future longitudinal studies are needed to support this hypothesis.

\section{Strengths and Limitations of the Study}

Strengths of the study include the multimodal imaging approach of combined gray matter volumetric analysis and white matter fiber tractography to assess the impact of structural integrity that promotes successful cognitive function in older adults. A robust tractography method was used. CSD overcomes the limitations of other DTI techniques, estimating the orientation of multiple intravoxel fiber populations in regions of white matter structures with crossing fibers like the fornix (Tournier et al., 2008; Jeurissen et al., 2011). The present study presents two methodological limitations; first, partial volume effects (PVE) affect DTI-based indices (Alexander et al., 2001; Szczepankiewicz et al., 2013). An individual voxel in brain imaging may contain different types of tissues; gray matter, white matter and cerebrospinal fluid. PVE refers to the impact that tissues, other than white matter, may have on tractography analysis, leading to underestimated FA values within a voxel [for review (Tohka, 2014)]. In order to attenuate PVE, we set the FA threshold to 0.2 , as a mean to eliminate all underestimated FA values. Second, concerns about the hippocampal subfield segmentation tool implemented in FreeSurfer were recently expressed when applied on T1weighted images with standard spatial resolution (Pluta et al., 2012; Wisse et al., 2014; de Flores et al., 2015; Yushkevich et al., 2015). Using the automated segmentation on images with $1 \mathrm{~mm}$-resolution may result in less accurate delineation of boundaries within subfields. It is thus recommended to acquire T2 images to improve automated subfield segmentation (Iglesias et al., 2015). Therefore, volumetric results from hippocampal subregions must be interpreted with caution. Nevertheless, we are confident that the automated segmentation in FreeSurfer provides highly useful information, allows for reproducible and - compared to manual segmentation less labor intensive and less biased results (Iglesias et al., 2015; Foo et al., 2017; Li et al., 2018; Zheng et al., 2018); 
and that individual subfields in our study were produced in the right position.

\section{CONCLUSION}

We investigated the effect of fornix FA and left DG volume on retrieval of episodic memory in healthy older adults. Our findings demonstrated that preserved fornix microstructure positively impacts on memory for false associations through a protective effect on left DG subfield volume. More generally, the results lend further support to the hypothesis that structural disconnection plays a crucial role in mediating deficits in the course of aging. Our study provides a neurobehavioral model for the linkage between structural memory network properties to explain inter-individual variability behavioral outcomes in older adults. Understanding this relationship is an important prerequisite for the development of interventions to counteract cognitive decline such as aerobic exercise, cognitive training or dietary interventions (Brickman et al., 2014; Antonenko et al., 2016b; Kobe et al., 2016).

\section{DATA AVAILABILITY STATEMENT}

The datasets generated during the current study are available from the corresponding author on reasonable request.

\section{ETHICS STATEMENT}

The studies involving human participants were reviewed and approved by the Charité - Universitätsmedizin Berlin, Corporate Member of Freie Universität Berlin, Humboldt-Universität

\section{REFERENCES}

Alexander, A. L., Hasan, K. M., Lazar, M., Tsuruda, J. S., and Parker, D. L. (2001). Analysis of partial volume effects in diffusion-tensor MRI. Magn. Reson. Med. 45, 770-780. doi: 10.1002/mrm.1105

Alexander, A. L., Lee, J. E., Lazar, M., and Field, A. S. (2007). Diffusion tensor imaging of the brain. Neurotherapeutics 4, 316-329.

Anblagan, D., Valdes Hernandez, M. C., Ritchie, S. J., Aribisala, B. S., Royle, N. A. I, Hamilton, F., et al. (2018). Coupled changes in hippocampal structure and cognitive ability in later life. Brain Behav. 8:e00838. doi: 10.1002/brb 3.838

Antonenko, D., Faxel, M., Grittner, U., Lavidor, M., and Floel, A. (2016a). Effects of transcranial alternating current stimulation on cognitive functions in healthy young and older adults. Neural Plast. 2016:4274127. doi: 10.1155/2016/427 4127

Antonenko, D., Hayek, D., Netzband, J., Grittner, U., and Flöel, A. (2019). tDCSinduced episodic memory enhancement and its association with functional network coupling in older adults. Sci. Rep. 9:2273. doi: 10.1038/s41598-01938630-7

Antonenko, D., Kulzow, N., Cesarz, M. E., Schindler, K., Grittner, U., and Floel, A. (2016b). Hippocampal pathway plasticity is associated with the ability to form novel memories in older adults. Front. Aging Neurosci. 8:61. doi: 10.3389/fnagi. 2016.00061

Antonenko, D., Meinzer, M., Lindenberg, R., Witte, A. V., and Floel, A. (2012). Grammar learning in older adults is linked to white matter microstructure and
Berlin, and Berlin Institute of Health, Department of Neurology, NeuroCure Clinical Research Center, Berlin, Germany. The patients/participants provided their written informed consent to participate in this study.

\section{AUTHOR CONTRIBUTIONS}

$\mathrm{DH}, \mathrm{DA}$, and AF designed the research. DH, FT, and DA analyzed the data. DH performed the tractography and volumetric analysis. DH prepared all figures. $\mathrm{DH}$ and $\mathrm{DA}$ wrote the manuscript. All authors reviewed and revised the manuscript.

\section{FUNDING}

This work was supported by the "Bundesministerium für Bildung und Forschung” (01GQ1424A).

\section{ACKNOWLEDGMENTS}

We thank Justus Netzband for help with data acquisition and Dr. Magdalene Ortmann for statistical support. This manuscript includes content from a Ph.D. thesis that is in line with the author's university policy, and can be accessed online (Hayek, 2019).

\section{SUPPLEMENTARY MATERIAL}

The Supplementary Material for this article can be found online at: https://www.frontiersin.org/articles/10.3389/fnagi. 2020.00079/full\#supplementary-material

functional connectivity. Neuroimage 62, 1667-1674. doi: 10.1016/j.neuroimage. 2012.05.074

Bakker, A., Kirwan, C. B., Miller, M., and Stark, C. E. (2008). Pattern separation in the human hippocampal CA3 and dentate gyrus. Science 319, 1640-1642. doi: $10.1126 /$ science. 1152882

Baron, R. M., and Kenny, D. A. (1986). The moderator-mediator variable distinction in social psychological research: conceptual, strategic, and statistical considerations. J. Pers. Soc. Psychol. 51, 1173-1182. doi: 10.1037/0022-3514.51. 6.1173

Bartzokis, G. (2004). Age-related myelin breakdown: a developmental model of cognitive decline and Alzheimer's disease. Neurobiol. Aging 25, 5-18. doi: 10. 1016/j.neurobiolaging.2003.03.001

Bartzokis, G. (2011). Alzheimer's disease as homeostatic responses to agerelated myelin breakdown. Neurobiol. Aging 32, 1341-1371. doi: 10.1016/j. neurobiolaging.2009.08.007

Basser, P. J., Pajevic, S., Pierpaoli, C., Duda, J., and Aldroubi, A. (2000). In vivo fiber tractography using DT-MRI data. Magn. Reson. Med. 44, 625-632. doi: 10.1002/1522-2594(200010)44:4<625::aid-mrm17>3.0 .co;2-o

Basser, P. J., and Pierpaoli, C. (1996). Microstructural and physiological features of tissues elucidated by quantitative-diffusion-tensor MRI. J. Magn. Reson. B 111, 209-219. doi: 10.1006/jmrb.1996.0086

Bennett, I. J., Huffman, D. J., and Stark, C. E. (2015). Limbic tract integrity contributes to pattern separation performance across the lifespan. Cereb. Cortex 25, 2988-2999. doi: 10.1093/cercor/bhu093 
Bennett, I. J., Stark, S. M., and Stark, C. E. L. (2018). Recognition memory dysfunction relates to hippocampal subfield volume: a study of cognitively normal and mildly impaired older adults. J. Gerontol. B Psychol. Sci. Soc. Sci. 74, 1132-1141. doi: 10.1093/geronb/gbx181

Breitenstein, C., Jansen, A., Deppe, M., Foerster, A. F., Sommer, J., Wolbers, T., et al. (2005). Hippocampus activity differentiates good from poor learners of a novel lexicon. Neuroimage 25, 958-968. doi: 10.1016/j.neuroimage.2004.12.019

Breitenstein, C., and Knecht, S. (2002). Development and validation of a language learning model for behavioral and functional-imaging studies. J. Neurosci. Methods 114, 173-179. doi: 10.1016/s0165-0270(01)00525-8

Brickman, A. M., Khan, U. A., Provenzano, F. A., Yeung, L.-K., Suzuki, W., Schroeter, H., et al. (2014). Enhancing dentate gyrus function with dietary flavanols improves cognition in older adults. Nat. Neurosci. 17:1798. doi: 10. 1038/nn.3850

Carballedo, A., Amico, F., Ugwu, I., Fagan, A. J., Fahey, C., Morris, D., et al. (2012). Reduced fractional anisotropy in the uncinate fasciculus in patients with major depression carrying the met-allele of the Val66Met brain-derived neurotrophic factor genotype. Am. J. Med. Genet. B Neuropsychiatr. Genet. 159B, 537-548. doi: 10.1002/ajmg.b.32060

Catani, M., Howard, R. J., Pajevic, S., and Jones, D. K. (2002). Virtual in vivo interactive dissection of white matter fasciculi in the human brain. Neuroimage 17, 77-94. doi: 10.1006/nimg.2002.1136

Dansokho, C., and Heneka, M. T. (2018). Neuroinflammatory responses in Alzheimer's disease. J. Neural Transm. 125, 771-779. doi: 10.1007/s00702-0171831-7

de Flores, R., La Joie, R., Landeau, B., Perrotin, A., Mezenge, F., de La Sayette, V., et al. (2015). Effects of age and Alzheimer's disease on hippocampal subfields: comparison between manual and FreeSurfer volumetry. Hum. Brain Mapp. 36, 463-474. doi: 10.1002/hbm.22640

De Shetler, N. G., and Rissman, J. (2017). Dissociable profiles of generalization/discrimination in the human hippocampus during associative retrieval. Hippocampus 27, 115-121. doi: 10.1002/hipo.22684

den Heijer, T., der Lijn, F., Vernooij, M. W., De Groot, M., Koudstaal, P. J., van der Lugt, A., et al. (2012). Structural and diffusion MRI measures of the hippocampus and memory performance. Neuroimage 63, 1782-1789. doi: 10 . 1016/j.neuroimage.2012.08.067

Douaud, G., Menke, R. A., Gass, A., Monsch, A. U., Rao, A., Whitcher, B., et al. (2013). Brain microstructure reveals early abnormalities more than two years prior to clinical progression from mild cognitive impairment to Alzheimer's disease. J. Neurosci. 33, 2147-2155. doi: 10.1523/JNEUROSCI.4437-12.2013

Douet, V., and Chang, L. (2014). Fornix as an imaging marker for episodic memory deficits in healthy aging and in various neurological disorders. Front. Aging Neurosci. 6:343. doi: 10.3389/fnagi.2014.00343

Doxey, C. R., and Kirwan, C. B. (2015). Structural and functional correlates of behavioral pattern separation in the hippocampus and medial temporal lobe. Hippocampus 25, 524-533. doi: 10.1002/hipo.22389

Duvernoy, H. M., Naidich, T., Fatterpekar, G. M., Raybaud, C., Risold, P. Y., Sakvolini, U., et al. (2005). The Human Hippocampus. Berlin: Springer.

Fischl, B., Salat, D. H., Busa, E., Albert, M., Dieterich, M., Haselgrove, C., et al. (2002). Whole brain segmentation: automated labeling of neuroanatomical structures in the human brain. Neuron 33, 341-355.

Fjell, A. M., Walhovd, K. B., Westlye, L. T., Ostby, Y., Tamnes, C. K., Jernigan, T. L., et al. (2010). When does brain aging accelerate? Dangers of quadratic fits in cross-sectional studies. Neuroimage 50, 1376-1383. doi: 10.1016/j.neuroimage. 2010.01.061

Fletcher, E., Raman, M., Huebner, P., Liu, A., Mungas, D., Carmichael, O., et al. (2013). Loss of fornix white matter volume as a predictor of cognitive impairment in cognitively normal elderly individuals. JAMA Neurol. 70, 13891395. doi: 10.1001/jamaneurol.2013.3263

Floel, A., Rosser, N., Michka, O., Knecht, S., and Breitenstein, C. (2008). Noninvasive brain stimulation improves language learning. J. Cogn. Neurosci. 20, 1415-1422. doi: 10.1162/jocn.2008.20098

Foo, H., Mak, E., Chander, R. J., Ng, A., Au, W. L., Sitoh, Y. Y., et al. (2017). Associations of hippocampal subfields in the progression of cognitive decline related to Parkinson's disease. Neuroimage Clin. 14, 37-42. doi: 10.1016/j.nicl. 2016.12.008

Frisoni, G. B., Ganzola, R., Canu, E., Rub, U., Pizzini, F. B., Alessandrini, F., et al. (2008). Mapping local hippocampal changes in Alzheimer's disease and normal ageing with MRI at 3 Tesla. Brain 131(Pt 12), 3266-3276. doi: 10.1093/brain/ awn280

Gazes, Y., Li, P., Sun, E., Razlighi, Q., and Tsapanou, A. (2018). Age specificity in fornix-to-hippocampus association. Brain Imaging Behav. 13, 1444-1452. doi: 10.1007/s11682-018-9958-1

Gondard, E., Chau, H. N., Mann, A., Tierney, T. S., Hamani, C., Kalia, S. K., et al. (2015). Rapid modulation of protein expression in the rat hippocampus following deep brain stimulation of the fornix. Brain Stimul. 8, 1058-1064. doi: 10.1016/j.brs.2015.07.044

Gorbach, T., Pudas, S., Lundquist, A., Oradd, G., Josefsson, M., Salami, A., et al. (2017). Longitudinal association between hippocampus atrophy and episodicmemory decline. Neurobiol. Aging 51, 167-176. doi: 10.1016/j.neurobiolaging. 2016.12.002

Grady, C. (2012). The cognitive neuroscience of ageing. Nat. Rev. Neurosci. 13, 491-505. doi: 10.1038/nrn3256

Hao, S., Tang, B., Wu, Z., Ure, K., Sun, Y., Tao, H., et al. (2015). Forniceal deep brain stimulation rescues hippocampal memory in Rett syndrome mice. Nature 526, 430-434. doi: 10.1038/nature15694

Hartopp, N., Wright, P., Ray, N. J., Evans, T. E., Metzler-Baddeley, C., Aggleton, J. P., et al. (2018). A key role for subiculum-fornix connectivity in recollection in older age. Front. Syst. Neurosci. 12:70. doi: 10.3389/fnsys.2018. 00070

Hayek, D. (2019). Association of Cognitive Performance with Hippocampal Network Integrity of Healthy Adults and its Modulation Through Non-Invasive Brain Stimulation. Ph. D thesis, Greifswald University, Greifswald.

Hayes, A. F., and Preacher, K. J. (2014). Statistical mediation analysis with a multicategorical independent variable. Br. J. Math. Stat. Psychol. 67, 451-470. doi: $10.1111 /$ bmsp. 12028

Head, D., Snyder, A. Z., Girton, L. E., Morris, J. C., and Buckner, R. L. (2005). Frontal-hippocampal double dissociation between normal aging and Alzheimer's disease. Cereb. Cortex 15, 732-739. doi: 10.1093/cercor/bhh174

Henson, R. N., Campbell, K. L., Davis, S. W., Taylor, J. R., Emery, T., Erzinclioglu, S., et al. (2016). Multiple determinants of lifespan memory differences. Sci. Rep. 6:32527. doi: 10.1038/srep32527

Iglesias, J. E., Augustinack, J. C., Nguyen, K., Player, C. M., Player, A., Wright, M., et al. (2015). A computational atlas of the hippocampal formation using ex vivo, ultra-high resolution MRI: application to adaptive segmentation of in vivo MRI. Neuroimage 115, 117-137. doi: 10.1016/j.neuroimage.2015.04.042

Insausti, R., and Amaral, D. (2004). The human nervous system. Hum. Nerv. Syst. 871-914.

Jeurissen, B., Leemans, A., Jones, D. K., Tournier, J. D., and Sijbers, J. (2011). Probabilistic fiber tracking using the residual bootstrap with constrained spherical deconvolution. Hum. Brain Mapp. 32, 461-479. doi: 10.1002/hbm. 21032

Kantarci, K. (2014). Fractional anisotropy of the fornix and hippocampal atrophy in Alzheimer's disease. Front. Aging Neurosci. 6:316. doi: 10.3389/fnagi.2014. 00316

Kerti, L., Witte, A. V., Winkler, A., Grittner, U., Rujescu, D., and Floel, A. (2013). Higher glucose levels associated with lower memory and reduced hippocampal microstructure. Neurology 81, 1746-1752. doi: 10.1212/01.wnl.0000435561. 00234.ee

Kesner, R. P., Lee, I., and Gilbert, P. (2004). A behavioral assessment of hippocampal function based on a subregional analysis. Rev. Neurosci. 15, 333-351.

Kobe, T., Witte, A. V., Schnelle, A., Lesemann, A., Fabian, S., Tesky, V. A., et al. (2016). Combined omega-3 fatty acids, aerobic exercise and cognitive stimulation prevents decline in gray matter volume of the frontal, parietal and cingulate cortex in patients with mild cognitive impairment. Neuroimage 131, 226-238. doi: 10.1016/j.neuroimage.2015.09.050

Kobe, T., Witte, A. V., Schnelle, A., Tesky, V. A., Pantel, J., Schuchardt, J. P., et al. (2017). Impact of resveratrol on glucose control. hippocampal structure and connectivity, and memory performance in patients with mild cognitive impairment. Front Neurosci. 11:105. doi: 10.3389/fnins.2017. 00105

Leemans, A., Jeurissen, B., Sijbers, J., and Jones, D. K. (2009). ExploreDTI: a graphical toolbox for processing, analyzing, and visualizing diffusion MR data. Proc. Intl. Soc. Mag. Reson. Med. 17:3537.

Lehrl, S. (2005). Manual Zum MWT-B. Balingen: Spitta-Verl. 
Li, W., Li, K., Guan, P., Chen, Y., Xiao, Y., Lui, S., et al. (2018). Volume alteration of hippocampal subfields in first-episode antipsychotic-naive schizophrenia patients before and after acute antipsychotic treatment. Neuroimage Clin. 20, 169-176. doi: 10.1016/j.nicl.2018.07.008

Lockhart, S. N., Mayda, A. B., Roach, A. E., Fletcher, E., Carmichael, O., Maillard, P., et al. (2012). Episodic memory function is associated with multiple measures of white matter integrity in cognitive aging. Front. Hum. Neurosci. 6:56. doi: 10.3389/fnhum.2012.00056

Lovblad, K. O., Schaller, K., and Vargas, M. I. (2014). The fornix and limbic system. Semin. Ultrasound CT MR 35, 459-473. doi: 10.1053/j.sult.2014.06.005

Ly, M., Adluru, N., Destiche, D. J., Lu, S. Y., Oh, J. M., Hoscheidt, S. M., et al. (2016). Fornix microstructure and memory performance is associated with altered neural connectivity during episodic recognition. J. Int. Neuropsychol. Soc. 22, 191-204. doi: 10.1017/S135561771500 1216

Lynn, S. K., and Barrett, L. F. (2014). Utilizing signal detection theory. Psychol. Sci. 25, 1663-1673. doi: 10.1177/0956797614541991

Marstaller, L., Williams, M., Rich, A., Savage, G., and Burianova, H. (2015). Aging and large-scale functional networks: white matter integrity, gray matter volume, and functional connectivity in the resting state. Neuroscience 290, 369-378. doi: 10.1016/j.neuroscience.2015.01.049

Metzler-Baddeley, C., Hunt, S., Jones, D. K., Leemans, A., Aggleton, J. P., and O'Sullivan, M. J. (2012). Temporal association tracts and the breakdown of episodic memory in mild cognitive impairment. Neurology 79, 2233-2240. doi: 10.1212/WNL.0b013e31827689e8

Metzler-Baddeley, C., Jones, D. K., Belaroussi, B., Aggleton, J. P., and O’Sullivan, M. J. (2011). Frontotemporal connections in episodic memory and aging: a diffusion MRI tractography study. J. Neurosci. 31, 13236-13245. doi: 10.1523/ JNEUROSCI.2317-11.2011

Metzler-Baddeley, C., Mole, J. P., Sims, R., Fasano, F., Evans, J., Jones, D. K., et al. (2019). Fornix white matter glia damage causes hippocampal gray matter damage during age-dependent limbic decline. Sci. Rep. 9:1060. doi: 10.1038/ s41598-018-37658-5

Mueller, S. G., and Weiner, M. W. (2009). Selective effect of age, Apo e4, and Alzheimer's disease on hippocampal subfields. Hippocampus 19, 558-564. doi: 10.1002/hipo.20614

Nyberg, L., Lövdén, M., Riklund, K., Lindenberger, U. and Bäckman, L. (2012). Memory aging and brain maintenance. Trends Cogn. Sci. 16, 292-305. doi: 10.1016/j.tics.2012.04.005

Oldfield, R. C. (1971). The assessment and analysis of handedness: the edinburgh inventory. Neuropsychologia 9, 97-113. doi: 10.1016/0028-3932(71)90 067-4

Pelletier, A., Periot, B., Dilharreguy, B., Hiba, M., Bordessoules, K., Peres, H., et al. (2013). Structural hippocampal network alterations during healthy aging: a multi-modal MRI study. Front. Aging Neurosci. 5:84. doi: 10.3389/fnagi.2013. 00084

Pereira, J. B., Valls-Pedret, C., Ros, E., Palacios, E., Falcon, C., Bargallo, N., et al. (2014). Regional vulnerability of hippocampal subfields to aging measured by structural and diffusion MRI. Hippocampus 24, 403-414. doi: 10.1002/hipo. 22234

Pluta, J., Yushkevich, P., Das, S. and Wolk, D. (2012). In vivo analysis of hippocampal subfield atrophy in mild cognitive impairment via semi-automatic segmentation of T2-weighted MRI. J. Alzheimers Dis. 31, 85-99. doi: 10.3233/ JAD-2012-111931

Powell, J. M., Rich, T. J. and Wise, E. K. (2016). Effectiveness of occupationand activity-based interventions to improve everyday activities and social participation for people with traumatic brain injury: a systematic review. Am. J. Occup. Ther. 70:7003180040p1-9.

Rolls, E. T. (2010). A computational theory of episodic memory formation in the hippocampus. Behav. Brain Res. 215, 180-196. doi: 10.1016/j.bbr.2010. 03.027

Rudebeck, S. R., Scholz, J., Millington, R., Rohenkohl, G., Johansen-Berg, H. and Lee, A. C (2009). Fornix microstructure correlates with recollection but not familiarity memory. J. Neurosci. 29, 14987-14992. doi: 10.1523/JNEUROSCI. 4707-09.2009

Sasson, E., Doniger, G. M., Pasternak, O., Tarrasch, R. and Assaf, Y. (2013). White matter correlates of cognitive domains in normal aging with diffusion tensor imaging. Front. Neurosci. 7:32. doi: 10.3389/fnins.2013.00032
Saunders, R. C. and Aggleton J. P. (2007). Origin and topography of fibers contributing to the fornix in macaque monkeys. Hippocampus 17, 396-411. doi: 10.1002/hipo.20276

Shing, Y. L., Rodrigue, K. M., Kennedy, K. M., Fandakova, Y., Bodammer, N., Werkle-Bergner, M., et al. (2011). Hippocampal subfield volumes: age, vascular risk, and correlation with associative memory. Front. Aging Neurosci. 3:2. doi: 10.3389/fnagi.2011.00002

Small, S. A., Chawla, M. K., Buonocore, M., Rapp, P. R. and Barnes, C. A. (2004). Imaging correlates of brain function in monkeys and rats isolates a hippocampal subregion differentially vulnerable to aging. Proc. Natl. Acad. Sci. U.S.A. 101, 7181-7186. doi: 10.1073/pnas.0400285101

Small, S. A., Schobel, S. A., Buxton, R. B., Witter, M. P. and Barnes, C. A. (2011). A pathophysiological framework of hippocampal dysfunction in ageing and disease. Nat. Rev. Neurosci. 12, 585-601. doi: 10.1038/nrn3085

Small, S. A., Tsai, W. Y., DeLaPaz, R., Mayeux, R. and Stern, Y. (2002). Imaging hippocampal function across the human life span: is memory decline normal or not? Ann. Neurol. 51, 290-295. doi: 10.1002/ana.10105

Stark, S. M., Yassa, M. A. and Stark, C. E. (2010). Individual differences in spatial pattern separation performance associated with healthy aging in humans. Learn Mem. 17, 284-288. doi: 10.1101/lm.1768110

Stark, S. M. and Stark, C. E. L. (2017). Age-related deficits in the mnemonic similarity task for objects and scenes. Behav. Brain Res. 333, 109-117. doi: 10.1016/j.bbr.2017.06.049

Szczepankiewicz, F., Latt, J., Wirestam, R., Leemans, A., Sundgren, P., van Westen Stahlberg, F., and Nilsson, M. (2013). Variability in diffusion kurtosis imaging: impact on study design, statistical power and interpretation. Neuroimage 76, 145-154. doi: 10.1016/j.neuroimage.2013.02.078

Tohka, J. (2014). Partial volume effect modeling for segmentation and tissue classification of brain magnetic resonance images: a review. World J. Radiol. 6, 855-864.

Toner, C. K., Pirogovsky, E., Kirwan, C. B. and Gilbert, P. E. (2009). Visual object pattern separation deficits in nondemented older adults. Learn. Mem. 16, 338-342. doi: 10.1101/lm.1315109

Tournier, J. D., Calamante, F., and Connelly, A. (2007). Robust determination of the fibre orientation distribution in diffusion MRI: non-negativity constrained super-resolved spherical deconvolution. Neuroimage 35, 1459-1472. doi: 10. 1016/j.neuroimage.2007.02.016

Tournier, J. D., Calamante, F., Gadian, D. G., and Connelly, A. (2004). Direct estimation of the fiber orientation density function from diffusion-weighted MRI data using spherical deconvolution. Neuroimage 23, 1176-1185. doi: 10. 1016/j.neuroimage.2004.07.037

Tournier, J. D., Yeh, C. H., Calamante, F., Cho, K. H., Connelly, A. and Lin, C. P. (2008). Resolving crossing fibres using constrained spherical deconvolution: validation using diffusion-weighted imaging phantom data. Neuroimage 42, 617-625. doi: 10.1016/j.neuroimage.2008.05.002

Walhovd, K. B., Westlye, L. T., Amlien, I., Espeseth, T., Raz, N., Salat, D. H., et al. (2011). Consistent neuroanatomical age-related volume differences across multiple samples. Neurobiol. Aging 32, 916-932. doi: 10.1016/j.neurobiolaging. 2009.05.013

Wilson, I. A., Gallagher, M., Eichenbaum, H., and Tanila, H. (2006). Neurocognitive aging: prior memories hinder new hippocampal encoding. Trends Neurosci. 29, 662-670. doi: 10.1016/j.tins.2006.10.002

Wisse, L. E., Biessels, G. J., and Geerlings, M. I. (2014). A critical appraisal of the hippocampal subfield segmentation package in freesurfer. Front. Aging Neurosci. 6:261. doi: 10.3389/fnagi.2014.00261

Wisse, L. E., Reijmer, Y. D., Kuijf, H. J., Leemans, A., Luijten, P. R., Koek, H. L., et al. (2015). Hippocampal disconnection in early Alzheimer's disease: a 7 tesla MRI study. J. Alzheimers Dis. 45, 1247-1256. doi: 10.3233/jad- 142994

Witt, J. A., Coras, R., Schramm, J., Becker, A. J., Elger, C. E., and Helmstaedter, C. (2014). The overall pathological status of the left hippocampus determines preoperative verbal memory performance in left mesial temporal lobe epilepsy. Hippocampus 24, 446-454. doi: 10.1002/hipo.22238

Yassa, M. A., Lacy, J. W., Stark, S. M., Albert, M. S., Gallagher, M., and Stark, C. E. (2011a). Pattern separation deficits associated with increased hippocampal CA3 and dentate gyrus activity in nondemented older adults. Hippocampus 21, 968-979. doi: 10.1002/hipo.20808

Yassa, M. A., Mattfeld, A. T., Stark, S. M., and Stark, C. E. (2011b). Age-related memory deficits linked to circuit-specific disruptions in the hippocampus. 
Proc. Natl. Acad. Sci. U.S.A. 108, 8873-8878. doi: 10.1073/pnas.110156 7108

Yesavage, J. A., Brink, T. L., Rose, T. L., Lum, O., Huang, V., Adey, M., et al. (1982). Development and validation of a geriatric depression screening scale: a preliminary report. J. Psychiatr. Res. 17, 37-49. doi: 10.1016/0022-3956(82) 90033-4

Yushkevich, P. A., Amaral, R. S., Augustinack, J. C., Bender, A. R., Bernstein, J. D., Boccardi, M., et al. (2015). Quantitative comparison of 21 protocols for labeling hippocampal subfields and parahippocampal subregions in in vivo MRI: towards a harmonized segmentation protocol. Neuroimage 111, 526-541. doi: 10.1016/j.neuroimage.2015. 01.004

Zamroziewicz, M. K., Paul, E. J., Zwilling, C. E., and Barbey, A. K. (2017). Predictors of memory in healthy aging: polyunsaturated fatty acid balance and fornix white matter integrity. Aging Dis. 8, 372-383. doi: 10.14336/AD.2017. 0501

Zatorre, R. J., Fields, R. D., and Johansen-Berg, H. (2012). Plasticity in gray and white: neuroimaging changes in brain structure during learning. Nat. Neurosci. 15, 528-536. doi: 10.1038/nn.3045

Zhang, R., Beyer, F., Lampe, L., Luck, T., Riedel-Heller, S. G., Loeffler, M., et al. (2018). White matter microstructural variability mediates the relation between obesity and cognition in healthy adults. Neuroimage 172, 239-249. doi: 10.1016/ j.neuroimage.2018.01.028

Zheng, F., Cui, D., Zhang, L., Zhang, S., Zhao, Y., Liu, X., et al. (2018). The volume of hippocampal subfields in relation to decline of memory recall across the adult lifespan. Front. Aging Neurosci. 10:320. doi: 10.3389/fnagi.2018. 00320

Zhuang, L., Sachdev, P. S., Trollor, J. N., Reppermund, S., Kochan, N. A., Brodaty, H., et al. (2013). Microstructural white matter changes, not hippocampal atrophy, detect early amnestic mild cognitive impairment. PLoS One 8:e58887. doi: /10.1371/journal.pone.0058887

Conflict of Interest: The authors declare that the research was conducted in the absence of any commercial or financial relationships that could be construed as a potential conflict of interest.

Copyright (C) 2020 Hayek, Thams, Flöel and Antonenko. This is an open-access article distributed under the terms of the Creative Commons Attribution License (CC BY). The use, distribution or reproduction in other forums is permitted, provided the original author(s) and the copyright owner(s) are credited and that the original publication in this journal is cited, in accordance with accepted academic practice. No use, distribution or reproduction is permitted which does not comply with these terms. 